\title{
Application of 3D tooth model for monitoring of implant space and inter-root distance without radiographs: A proof of concept study
}

\section{Mirinae Park}

Chonnam National University School of Dentistry

Kyung-Min Lee ( $\nabla$ ortholkm@jnu.ac.kr)

Chonnam National University School of Dentistry https://orcid.org/0000-0003-3102-4550

\section{Methodology}

Keywords: 3D Tooth model, CBCT, intraoral scan, root position, radiation

Posted Date: April 7th, 2020

DOI: https://doi.org/10.21203/rs.3.rs-20887/v1

License: (c) (1) This work is licensed under a Creative Commons Attribution 4.0 International License.

Read Full License 


\section{Abstract}

Background Radiographs are integral for evaluating of implant space and inter-root distance. The purpose of this report is to introduce a method for evaluating 3D root position with minimal radiation using 3D tooth model which is composed of intraoral-scanned crown and CBCT-scanned root.

Materials and methods An intraoral scan and CBCT scan of the patient were obtained before treatment. In the CBCT image, tooth segmentation was performed by isolating individual tooth from the maxillary and mandibular alveolar bone using software program. By combining segmented individual tooth with the intraoral scan, consequently, 3D tooth model was fabricated.

Results After treatment, the additional intraoral scan at post-treatment was integrated into the tooth model and the resulting position of the root could be predicted without additional radiographs. It is possible to monitor the root position using 3D tooth model without the need for additional radiographs after a pretreatment $\mathrm{CBCT}$ scan.

Conclusion The application of 3D tooth model benefits the patient by reducing radiation exposure while providing the clinician with a precise treatment evaluation for monitoring tooth movement.

\section{Background}

A successful treatment depends not only on initial diagnosis but also on accurate assessment of treatment progress. For monitoring and evaluation of tooth movement, especially, root movement, clinicians need to take cone-beam computed tomography (CBCT) scan. As CBCT can depict the true root position and angulation in 3-dimensions, accurate evaluation of tooth movement is possible with the help of CBCT scan. However, it is challenging for clinicians to take the repeated CBCT scan because of patients' or their parents' concern on radiation hazard. Although the radiation in dental imaging is far less compared to the imaging in medicine, multiple CBCT scan is not recommended clinically due to the public's increasing concerns on radiation hazard. ${ }^{1}$

The failure to sequential assessment of the root position leads to unexpected treatment time delay and undesired results. The purpose of this report was to suggest a method for monitoring of threedimensional (3D) tooth movement without additional CBCT scans. The method uses 3D tooth models composed of intraoral-scanned crown and CBCT-scanned root. Intraoral scan and CBCT scan of the patient were obtained before treatment. In the CBCT image, the tooth was isolated from the alveolar bone using software program. By combining isolated individual tooth and the intraoral scan, tooth models which were composed of intraoral-scanned crown and CBCT-scanned root were fabricated. When evaluation of root position is needed during treatment, the patient's dentition is scanned using an intraoral scanner. Additional intraoral scan at any stage of treatment is integrated into the tooth models and its resulting position of the root is predicted without the need of CBCT scan. Based on the tooth models, clinicians can evaluate 3D tooth movement during treatment with only intraoral scan and without additional CBCT scans. The purpose of this report is to introduce a method for evaluating 3D root 
position with minimal radiation using 3D tooth model which is composed of intraoral-scanned crown and CBCT-scanned root.

\section{Materials And Methods}

A 23-year-old female patient visited the department of orthodontics at Chonnam National University with the chief complaint of midline discrepancy. Intraoral photographs showed a midline discrepancy and retained left deciduous canine. On the pretreatment records including lateral cephalogram and panoramic radiographs, congenital missing of bilateral maxillary lateral incisors was observed and left deciduous canine was retained at the canine area. CBCT (Alphard Vega; Asahi Roentgen, Kyoto, Japan; $80 \mathrm{kV}$ and 5 $\mathrm{mA}$; voxel size, $0.39 \mathrm{~mm} \times 0.39 \mathrm{~mm} \times 0.39 \mathrm{~mm}$; and field of view, $200 \mathrm{~mm} \times 179 \mathrm{~mm}$ ) was taken in the department of prosthodontics for evaluation of available space for implants. After treatment planning with prosthodontist, space opening for prosthetic restoration of maxillary lateral incisors was decided. Considering the root positions of maxillary central incisors and canines, the space opening was planned to the right lateral incisor area and left canine area. For the right side, the canine would be retracted to gain available space for implant of right lateral incisor, and, for the left side, lateralization of canine and space gaining on the canine area was planned. After 9-month of orthodontic treatment, the patient was referred to the department of prosthodontics for evaluation of implant spaces. Prosthodontist evaluated the implant space using panoramic radiograph and suggested to gain more space for left implant area (Fig. 1). It is believed that the ideal distance from the base of the contact point to the alveolar bone crest between a tooth and an implant is $3 \mathrm{~mm}$ to $5 \mathrm{~mm}^{2,3}$. However, panoramic radiograph may be inaccurate in showing the true root positions. Previous researches have shown that panoramic radiograph contains inherent image distortions, and the assessment of root parallelism using panoramic radiography should be performed with caution, especially in premolar extraction sites ${ }^{4,5}$. Thus, additional CBCT scan was needed for accurate evaluation of the space for lateral incisor and canine, but the patient's radiation exposure was concerned.

\section{Fabrication of 3D Tooth Model at pretreament}

Intraoral scan and CBCT scan of the pretreatment record was used to fabricate the tooth models. Intraoral scan on the maxillary and mandibular arches were obtained using TRIOS scanner (3Shape, Copenhagen, Denmark) and scan data were converted into stereolithography (STL) format on the OrthoAnalyzer ${ }^{\mathrm{TM}}$ software (3Shape). The CBCT scan data was imported into InVivo5 software (version 5.1, Anatomage, San Jose, CA) for tooth segmentation. In the "MD (medical design) studio" module, tooth was isolated from alveolar bone and was segmented individually using the "sculpt" function and converted into STL file format.

Segmented tooth including root from CBCT scan and intraoral scan data were imported into Rapidform ${ }^{\mathrm{TM}}$ 2006 (3D Systems, RockHill, SC). CBCT scan and intraoral scan data were integrated using registration function of the software. Initial registration was performed by selecting more than three corresponding 
points of each image, resulting in a rough alignment. Then a "fine" automatic best-fit registration was used to finalize the matches. In order to fabricate the tooth model composed of intraoral scanned crown and CBCT-scanned root, the area of CBCT-scanned crown was removed from the integrated image and finally the intraoral scanned crown and CBCT-scanned root were merged using the function of the program.

\section{Results}

After the consultation with prosthodontist, the patient's maxillary left quadrant area was scanned using intraoral scanner. This intraoral scan data at midtreatment (9-month of orthodontic treatment) was registered onto the tooth models at pretreatment. The resulting position of the root could be predicted without additional CBCT scan for visualization and evaluation of distance between the roots after treatment (Fig 2a). The space seemed too close between the fixture and the adjacent roots in the panorama, however, there was enough space for a fixture. The space was $8.6 \mathrm{~mm}$ at midroot level and $11.6 \mathrm{~mm}$ at apex level (Fig $2 \mathrm{~b}$ ). This indicated that more space regaining was not needed. Patient could start prosthetic restoration treatment without additional orthodontic treatment. Without additional CBCT scans, 3D root positions were evaluated and unnecessary additional treatment and resulting undesired delay of treatment period were prevented. After 2 years of post-treatment, the intraoral photographs and panoramic radiograph showed that the implants were stable with good esthetic and occlusal results.

\section{Discussion}

Integrating intraoral scan and $\mathrm{CBCT}$ image by replacing the dental part of a CT image with an intact dental image allows precise evaluation of 3D tooth movement in clinical applications where 3D information of root is needed including tooth arrangement, tooth movement monitoring, and orthodontic treatment simulation. CBCT images contain artifacts because of beam hardening and enamel also creates an artifact ${ }^{6}$, which can appear because of differences in the radiation attenuation coefficient of the natural dentition without metal restoration. These artifacts may influence the accuracy of integration when superimposing the CBCT and intraoral scans. As CВCT image is incapable of accurately representing occlusion, in particular, the occlusal surface in dental images ${ }^{7}$, replacing the dental part of a CT image with an intact dental image is essential for accurate fabrication of 3D tooth models ${ }^{8,9}$. In this report, our method first fabricated the tooth models at pretreatment by merging CBCT and intraoral scanned crown at pretreatment and then, this was used for sequential evaluation of tooth movement. When integrating intraoral scan and $\mathrm{CBCT}$ image, an accurate registration of the two images is essential for the high quality of integrated image. Tooth segmentation process of separating individual tooth from the alveolar bone on the CBCT images is a fundamental step for fabrication of tooth model. Many thirdparty software programs have been used for imaging processing. The need of advanced program for accurate tooth segmentation/isolation is required for future researches. Individual tooth models can be used to guide the clinicians to anticipated root movement. This technique gives us a way to look at 3D tooth movement and may give us better predictability. The application of 3D tooth model benefits the 
patient by reducing radiation exposure while providing the clinician with a precise treatment evaluation for monitoring tooth movement.

\section{Conclusion}

The application of 3D tooth model benefits the patient by reducing radiation exposure while providing the clinician with a precise treatment evaluation for monitoring tooth movement.

\section{Declarations}

\section{Ethics approval and consent to participate}

Ethical approval: All procedures performed in study involving human participant were in accordance with the ethical standards of the institutional and national research committee (Institutional Review Board of the Chonnam National University Dental Hospital, Gwangju, Korea; CNUDH-EXP-2019-019) and with the 1964 Helsinki declaration and its later amendments or comparable ethical standards.

Informed consent: For this type of study, formal consent is not required. Data was provided by the institutions as anonymized files and the methodology was accepted by the relevant ethical committee.

\section{Consent for publication}

Not applicable.

\section{Availability of data and materials}

The datasets included during the current study are available from the corresponding author on reasonable request.

\section{Competing interests}

Mirinae Park declares that she has no conflict of interest. Author Kyung-Min Lee declares that she has no conflict of interest.

\section{Funding}

This research was supported by Basic Science Research Program through the National Research Foundation of Korea (NRF) funded by the Ministry of Science, ICT \& Future Planning (NRF2014R1A1A1003559 and NRF-2017R1D1A1B03032132). The Funding body will pay the publication charge.

\section{Authors' contributions}


MP wrote the manuscript and KML designed and wrote the manuscript. All authors read and approved the final manuscript.

\section{Acknowledgements}

No

\section{References}

1. Ludlow JB, Davies-Ludlow LE, Brooks SL, Howerton WB. Dosimetry of 3 CBCT devices for oral and maxillofacial radiology: CB Mercuray, NewTom $3 G$ and i-CAT. Dentomaxillofac Radiol 2006;35:21926.

2. Thilander B, Odman J, Jemt T. Single implants in the upper incisor region and their relationship to the adjacent teeth. An 8-year follow-up study. Clin Oral Implants Res 1999;10:346-55.

3. Gastaldo JF, Cury PR, Sendyk WR. Effect of the vertical and horizontal distances between adjacent implants and between a tooth and an implant on the incidence of interproximal papilla. J Periodontol 2004;75:1242-6.

4. Mckee IW, Glover KE, Williamson PC, Lam EW, Heo G, Major PW. The effect of vertical and horizontal head positioning in panoramic radiography on mesiodistal tooth angulations. Angle Orthod 2001;71:442-51.

5. Garcia-Figueroa MA, Raboud DW, Lam EW, Heo G, Major PW. Effect of buccolingual root angulation on the mesiodistal angulation shown on panoramic radiographs. Am J Orthod Dentofacial Orthop 2008;134:93-9.

6. Schulze R, Heil U, Gross D, Bruellmann DD, Dranischnikow E, Schwanecke U et al. Artefacts in CBCT: a review. Dentomaxillofac Radiol 2011;40:265-73.

7. Santler G, Kärcher H, Ruda C. Indications and limitations of three-dimensional models in craniomaxillofacial surgery. J Craniomaxillofac Surg 1998;26:11-6.

8. Noh H, Nabha W, Cho JH, Hwang HS. Registration accuracy in the integration of laser-scanned dental images into maxillofacial cone-beam computed tomography images. Am J Orthod Dentofacial Orthop 2011;140:585-91.

9. Sun LJ, Hwang HS, Lee KM. Registration area and accuracy when integrating laser-scanned and maxillofacial cone-beam computed tomography images. Am J Orthod Dentofacial Orthop 2018;153:355-61.

\section{Figures}




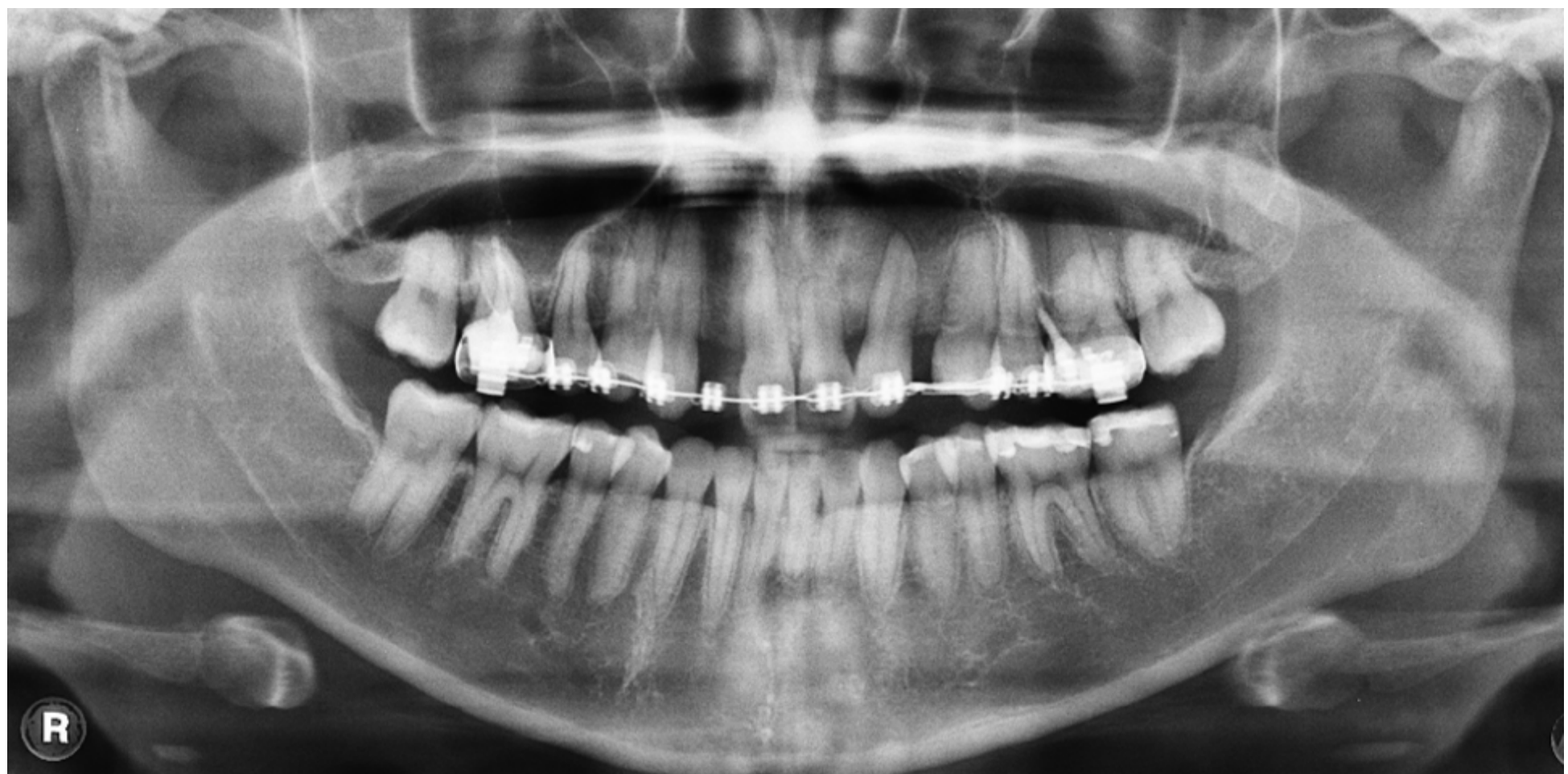

Figure 1

Panoramic radiographs after 9-month of orthodontic treatment.

3D Tooth Models at pretreatment

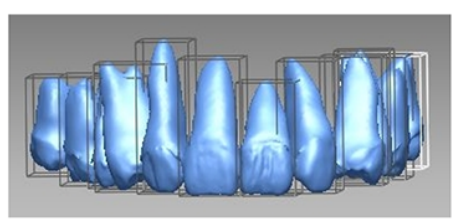

Intraoral scan at midtreatment

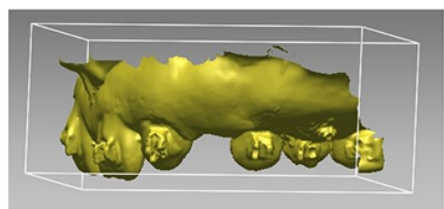

$\checkmark$

Tooth Models with expected root position

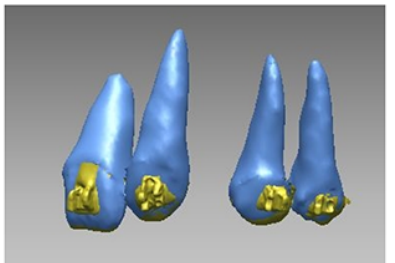

a
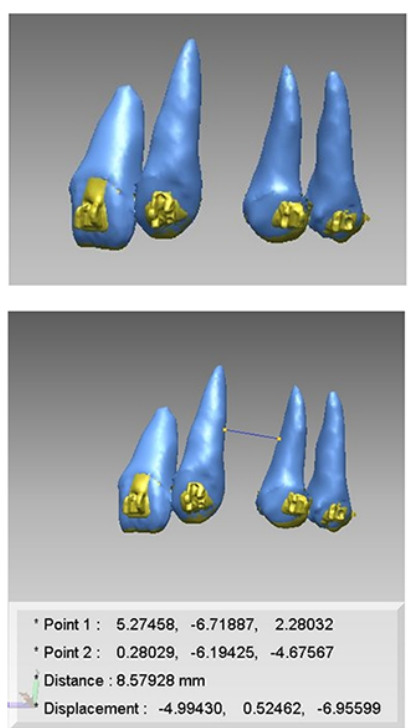

b
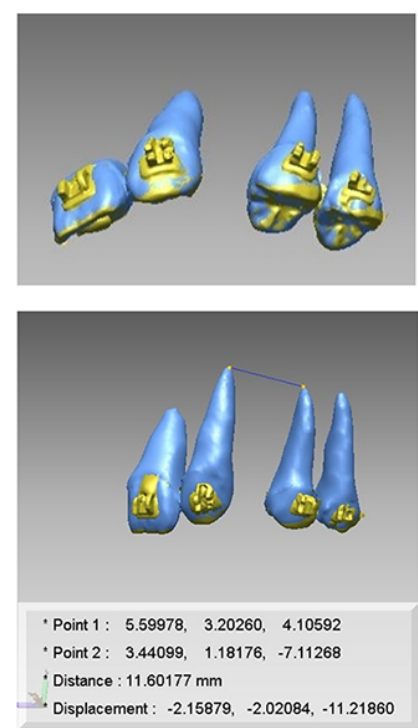

Displacement: $-2.15879,-2.02084,-11.21860$

\section{Figure 2}

Generation of tooth model with expected root position using tooth model at pretreatment and intraoral scan at midtreatment. Intraoral scan at midtreatment was registered onto the individual tooth model at pretreatment (a) and the root distance was measured in the program (b). 